This is a self-archived version of an original article. This version may differ from the original in pagination and typographic details.

Author(s): Selenius, Elli; Malola, Sami; Häkkinen, Hannu

Title: Analysis of the plasmonic excitations in assemblies of three-dimensional electron clusters

Year: 2020

Version: Published version

Copyright: (C2020 American Physical Society

Rights: In Copyright

Rights url: http://rightsstatements.org/page/InC/1.0/?language=en

Please cite the original version:

Selenius, E., Malola, S., \& Häkkinen, H. (2020). Analysis of the plasmonic excitations in assemblies of three-dimensional electron clusters. Physical Review B, 102(19), Article 195433. https://doi.org/10.1103/physrevb.102.195433 


\title{
Analysis of the plasmonic excitations in assemblies of three-dimensional electron clusters
}

\author{
Elli Selenius $\odot$ and Sami Malola \\ Department of Physics, Nanoscience Center, University of Jyväskylä, FI-40014 Jyväskylä, Finland \\ Hannu Häkkinen $\odot^{*}$ \\ Departments of Physics and Chemistry, Nanoscience Center, University of Jyväskylä, FI-40014 Jyväskylä, Finland
}

(Received 10 August 2020; revised 9 November 2020; accepted 11 November 2020; published 30 November 2020)

\begin{abstract}
In the quest to built novel metamaterials with unique optical properties, three-dimensional assemblies of metal clusters and nanoparticles are gathering significant attention. Organized geometries, such as tetrahedra and icosahedra, can be built, for example, by using DNA strands or virus capsids as templates. Here we use the jellium model and time-dependent density functional theory to study the plasmonic resonances in different arrangements of eight-electron clusters from the electronic perspective. A charge transfer ratio index based on the induced transition densities is used to quantify the charge transfer nature of the excitations at different energies. We vary the size, shape, and intercluster separation, building systems of four-cluster tetrahedra, 12-cluster icosahedra and cuboctahedra, and 20-cluster dodecahedra. All the studied systems are found to have charge transfer plasmon-type excitations at low energies. Analysis of the electron-hole transitions contributing to the transition dipole moment is further used to characterize these excitations, showing that they have significant screening contributions unlike the higher-energy excitations. The understanding gained for the optical response of these simple model systems can help in interpreting the properties of real, complex cluster systems.
\end{abstract}

DOI: 10.1103/PhysRevB.102.195433

\section{INTRODUCTION}

Since the coupling of the plasmonic excitations in adjacent metal clusters or nanoparticles (NPs) changes the optical response [1], assembly of such clusters or NPs is a way to create materials with novel optical properties. The creation, characterization, and application of these metamaterials, such as three-dimensional (3D) assemblies or aggregates of plasmonic NPs, has received significant attention in recent years $[2,3]$.

Single metal clusters or nanoparticles can support localized surface plasmon resonances (LSPR), which are collective oscillations of the conduction electrons on the surface of the clusters. In dimers or other cluster assemblies, these plasmon peaks can couple to form a bonding combination of the individual plasmons [1]. Clusters or NPs that have a sufficiently small separation can also support charge transfer plasmons (CTPs), which involve the electron density oscillating between the individual clusters [4].

Three-dimensional arrangements of plasmonic NPs can be assembled, for example, by using DNA templates [5,6], manipulation with atomic force microscope [7], tethering or coating the clusters with block copolymers [8-10], or using viruses or virus-like particles as templates [11-13]. The geometries achieved include among others spherical vesicles [10], tetrahedra [5-9], icosahedra [9,11], and other arrangements with fivefold symmetries [12,13].

Spherical gold NPs in tetrahedral arrangements were studied theoretically by Urzhumov et al. [14] using the plasmon hybridization (PH) model [1] and finite element frequency

*hannu.j.hakkinen@jyu.fi domain calculations. They proposed the concept of metaflu$i d s$, which are fluids containing these plasmonic metaclusters. Using the PH model and Drude theory, they found the plasmon resonance energies dependent on the separation of the clusters.

One application for these plasmonic assemblies or "plasmonic molecules" is surface-enhanced Raman scattering (SERS). Via the creation of hot spots between the plasmonic NPs, these assemblies were found to enhance the signal compared to single NPs $[8,10,12,13]$. Pazos-Perez et al. fabricated several 2D and 3D geometries of block copolymer-protected Au NPs using evaporation of emulsion droplets [8]. The NPs in the arrangements had a diameter of approximately $50 \mathrm{~nm}$ and interparticle separations of around $2 \mathrm{~nm}$. A big jump in the SERS enhancement was observed going from planar geometries to the tetrahedron. In the case of the plasmonic vesicles, Huang et al. manufactured structures of several hundreds of nanometers using different block copolymers as ligands and gold NPs with diameters of 20, 50, and $80 \mathrm{~nm}$ [10]. The assembly of the NPs was also achieved by subsequent emulsification and evaporation, and the separations between clusters varied from $0.5 \mathrm{~nm}$ for the largest clusters to $11.6 \mathrm{~nm}$ for some of the vesicles of the smallest clusters. They measured the largest enhancements for the largest used Au NPs, proposing that this might be due to the smallest intercluster separations in these structures.

Arrangements of plasmonic nanoclusters can also be used in light-assisted drug delivery [15]. The release of cargo can be achieved, for example, by embedding metal NPs in the outer layers of microcapsules [16] or coating loaded liposomes with metal NPs [17]. In these techniques, the plasmon resonance of the metal NPs is used to convert light into 
thermal energy, damaging the capsule or liposome and allowing the material inside to exit.

In addition to larger nanoparticles, assemblies can also be built of atomically precise clusters. Nonappa et al. have fabricated spherical, hollow capsids with a diameter of around $200 \mathrm{~nm}$ consisting of $\mathrm{Au}_{102}-p \mathrm{MBA}_{44}(p \mathrm{MBA}=p$ mercaptobenzoic acid) clusters [18]. The self-assembly of the clusters in a water-methanol mixture seems to be guided by the balance of protonated and deprotonated carboxylic acids in the ligands. Marjomäki et al. used $\mathrm{Au}_{102}-p \mathrm{MBA}_{44}$ clusters to coat enteroviruses with tens of clusters [19]. The clusters were functionalized with maleimide linkers to attach to specific sites in the virus capsid. In the study the clusters were used as labels for transmission electron microscopy, but the authors suggested also using the geometry- and separationdependent optical properties of the clusters for spectroscopy of the viruses and manipulation of the virus capsid by electromagnetic radiation. The clusters used in these two studies were not plasmonic, but similar techniques could be used to built plasmonic cluster assemblies.

To understand the plasmonic coupling and the optical properties in different cluster assemblies from the electronic perspective, we use the jellium model and density functional theory (DFT) to study geometric arrangements of eight-electron clusters with a tetrahedral, icosahedral, cuboctahedral, and dodecahedral shape. We have previously studied the LSPR in single clusters and bonding dipolar plasmons in planar assemblies [20] and CTPs in dimers [21] using the same methods. The simple jellium model enables us to concentrate on the electronic response and to handle large systems with lower computational cost than with fully atomistic models. In addition, we have seen that assemblies of even the small eight-electron jellium clusters can support collective excitations such as CTPs and bonding combinations of the LSPRs.

Since there is a spherical shell closing for eight electrons in the jellium model, we use a positive background in the shape of a sphere for the clusters. This spherical symmetry of the individual clusters also simplifies the analysis of the different cluster assemblies, making it easier to observe the effect of the geometry of the arrangement. For real clusters, the atomic structure obviously prevents the shape of the eight-electron sodium cluster from being exactly spherical. In atomistic $a b$ initio calculations, $\mathrm{Na}_{8}$ is found to prefer a shape with the $\mathrm{T}_{d}$ point group, higher in symmetry than the neighboring cluster sizes [22,23].

Zhang et al. have showed that for dimers of sodium clusters with about 300 atoms, the atomic structure affects the exact energies of the main absorption peaks and the maximum separation at which the CTP peak is manifested [24]. However, the main trends for the absorption spectra were reproduced also by the jellium model. The shape of $\mathrm{Na}_{8}$ differs more from a sphere than the shape of these larger clusters, but we can still expect that the jellium assemblies can qualitatively reproduce results of the corresponding atomic systems.

Classical simulations have been previously performed for three-dimensional cluster arrangements with clusters the size of tens of nanometers $[5,7-10,12,14]$. Unlike the LSPR-based plasmons, the tunneling CTPs present at small separations cannot be modeled using classical approaches [4]. Since our (a)

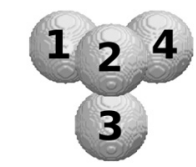

(c)

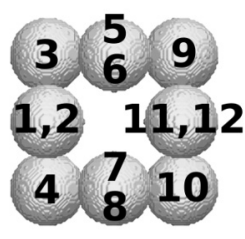

(b)

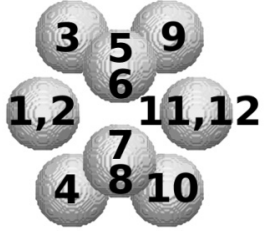

(d)

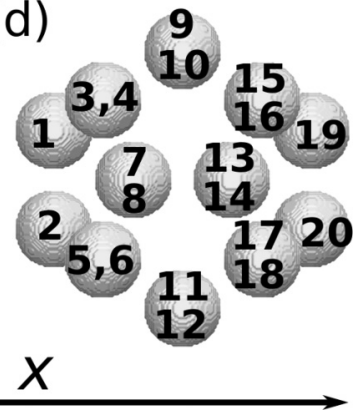

FIG. 1. The jellium backgrounds for the studied geometries: (a) tetrahedron, (b) icosahedron, (c) cuboctahedron, and (d) dodecahedron. The clusters are numbered for further reference. The direction of the optical analysis $(x)$ is indicated.

methods and systems allow us to reach the CTP region, we will focus more on these less studied excitations.

The analyzed cluster assemblies have been chosen to be shell-like or capsid-like to mimic the experimental setups with capsids of clusters or clusters or NPs arranged on a virus. In the experiments thus far, either the capsid or the NPs have mostly been far bigger than the systems studied here. However, our jellium systems serve as smaller-scale models to study the optical phenomena of cluster systems with subnanometer gaps in detail, using the methods and tools that we have already developed for these jellium clusters. In the future, our analysis can help to interpret the more complex optical responses of real, atomistic cluster systems.

\section{THEORY AND METHODS}

The studied systems consist of spherical eight-electron clusters, arranged in a hollow capsid-like formation. Tetrahedral, icosahedral, cuboctahedral, and dodecahedral symmetry were used, with $4,12,12$, and 20 clusters, respectively. The clusters were placed on the vertices of these shapes. The density of sodium was used for the jellium background, resulting in a radius of $r=4.1 \AA$ for one cluster. The largest system, dodecahedron, had an intercluster separation of $S=1.0 \AA=$ $0.25 r$. For the other geometries, two different separations were used: $S=1.0 \AA$ and $S=2.1 \AA=0.5 r$. The jellium systems can be seen in Fig. 1.

The DFT calculations were performed with the real-space grid GPAW program $[25,26]$, which allowed us to easily construct the systems. Because of the uniform background, the LDA functional [27] was chosen for the exchange correlation. In the case of charge transfer between clusters, LDA can reproduce also charge transfer plasmons (see the supporting information of Ref. [21]). The optical spectrum was calculated 
(a)

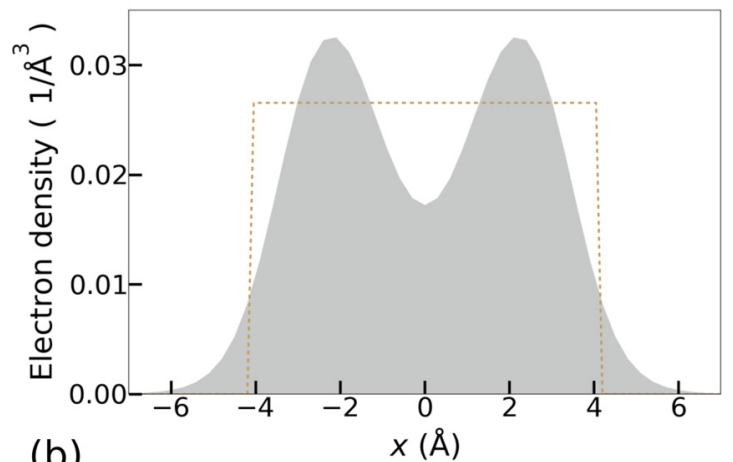

(b)

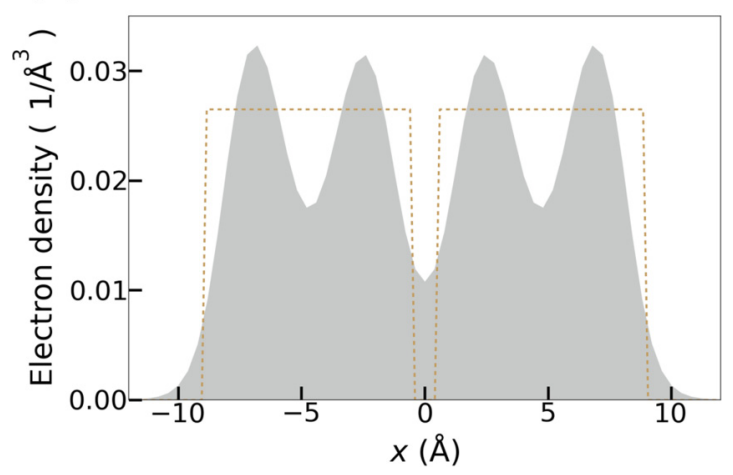

FIG. 2. The electron density (in gray) along the $x$ axis for (a) a single eight-electron cluster and (b) a dimer of two eight-electron clusters with a separation of $S=0.25 r$. The $x$ axis is the dimer axis. The positive jellium background density is indicated with a dashed line.

using the linear response time-dependent DFT (lr-TDDFT) as implemented in GPAW [28].

In GPAW, the jellium background is constructed by assigning the positive background charge to certain gridpoints. For the spherical jellium clusters, these are the gridpoints with coordinates $\mathbf{r}$ for which

$$
\left|\mathbf{r}-\mathbf{R}_{\mathbf{0}}\right|<N^{1 / 3} r_{s},
$$

where $\mathbf{R}_{\mathbf{0}}$ is the center of the cluster, $N$ is the number of electrons of the cluster, and $r_{s}$ is the Wigner-Seitz radius of sodium. We used the value $r_{s}=2.08 \AA$. Since the charge is divided uniformly, inside the jellium spheres the background density is approximately

$$
\rho_{J}=\left(\frac{4}{3} \pi r_{s}^{3}\right)^{-1} \approx 0.0265 \AA^{-3} .
$$

This positive density is then added to the electron density when calculating the Hartree potential, which contains the Coulombic repulsion and attraction of the charges. The selfconsistently calculated electron density is not uniform but experiences oscillations and spill-out from the positive background.

To illustrate the extent of this spill-out of electrons and the electron density overlap for adjacent clusters, the electron density along $x$ axis for a single cluster and dimer are shown in gray in Fig. 2. The uniform positive background density $\rho_{J}$ is indicated with a dashed line. The dimer has a separation of $S=0.25 r$. The electron density for a dimer of a separation of $S=0.5 r$ can be seen in the supporting information of Ref. [21], where the effect of the electron density overlap on the CTPs is discussed for dimers. Figure 2(a) shows that the electron density tail extends about $2 \AA$ outside of the jellium surface. For clusters with a separation of $S=0.25 r=1.0 \AA$, there is significant electron density between the clusters, as can be seen from Fig. 2(b). For the separation of $S=0.5 r=2.1 \AA$, the electron density overlap is smaller but non-negligible.

The calculations were performed at $0 \mathrm{~K}$ in vacuum, and a minimum of approximately $8 \AA$ of empty space was left between the jellium edges and the edge of the calculation box. Because of the uniform jellium background, a relatively large grid spacing of $h=0.4 \AA$ was employed. Testing the convergence with respect to the grid spacing, the spectrum of one of the tetrahedral systems was found to be the same for $h=0.3 \AA$ and $h=0.4 \AA$. For the optical spectrum calculation, the used energy cutoff was $7 \mathrm{eV}$ for the tetrahedra, $6 \mathrm{eV}$ for the icosahedra and cuboctahedra, and $5 \mathrm{eV}$ for the dodecahedron.

Selected peaks of the spectra were also further analyzed using the time-dependent density functional perturbation theory [29], where the system is excited with a cosinoidal laser pulse. This allows us to separate the contributions from different Kohn-Sham electron-hole transitions to the transition dipole moment and also to calculate the induced density. Here we use a laser pulse polarized in the $x$ direction, as indicated in Fig. 1. We visualized these contributions employing the dipole transition contribution map (DTCM) scheme [30,31]. This was combined with the $\mathrm{Y}_{l m}$ analysis [32], where the Kohn-Sham orbitals of the clusters are projected into spherical harmonics to show the superatomic symmetries. This analysis was done for each spherical eight-electron cluster separately, and the contributions were summed for the total system.

To have a quantitative measure of the charge transfer character of an excitation, we have employed an index, the charge transfer ratio (CTR). In our previous study of dimeric systems [21], we employed a similar index which was calculated using the dipole moment of the charge transfer and the induced dipole moment of the system. Now, due to the more complicated symmetries of the systems, we employ an alternative way to calculate a similar index. We want to compare the ratio of the total induced charge momentarily transferred between clusters at the studied excitation energy to the total induced charge oscillating in the whole system.

Due to symmetry, the clusters that have a center at the $x=0$ plane have a total of zero induced charge, and the clusters at the left (right) all have either a positive or negative charge. Thus, we can have a measure of the total charge transfer between clusters by summing the induced charge for all the clusters on the left. To normalize, we divide by the total positive induced charge in the whole system, or half of the integral of the absolute value of the induced density. Let us use the index $i$ for the clusters in one system, and divide the calculation box to the left and right sides. Now we can write the CTR index as

$$
\mathrm{CTR}=\frac{\left|\sum_{i \text { in left }} \int_{\text {cluster } i} \rho_{\text {ind }}(\mathbf{r}) d \mathbf{r}\right|}{\int_{\text {all }}\left|\rho_{\text {ind }}(\mathbf{r})\right| / 2 d \mathbf{r}},
$$


TABLE I. The binding energy per cluster and HOMO-LUMO gap for all the studied systems in units of $\mathrm{eV}$. The abbreviations used in the text are also shown. The values for two dimer systems consisting of spherical eight-electron clusters are included for comparison.

\begin{tabular}{lllcc}
\hline \hline Abbreviation & \multicolumn{1}{c}{ Shape } & $S / r$ & $E_{b} /$ cluster & H-L gap \\
\hline Th1 & Tetrahedron & 0.5 & 0.073 & 1.17 \\
Th2 & Tetrahedron & 0.25 & 0.19 & 0.98 \\
Ih1 & Icosahedron & 0.5 & 0.12 & 1.11 \\
Ih2 & Icosahedron & 0.25 & 0.32 & 0.82 \\
Ch1 & Cuboctahedron & 0.5 & 0.098 & 1.12 \\
Ch2 & Cuboctahedron & 0.25 & 0.26 & 0.80 \\
Dh & Dodecahedron & 0.25 & 0.20 & 0.92 \\
- & Dimer & 0.5 & 0.023 & 1.27 \\
- & Dimer & 0.25 & 0.066 & 1.13 \\
\hline \hline
\end{tabular}

where $\rho_{\text {ind }}(\mathbf{r})$ is the induced density and the summation goes over all the clusters on the left side of the system. For example, for the icosahedron visualized in Fig. 1(b), these clusters are numbered 1-4. The integral in the numerator is performed over the volume assigned for each cluster. The division of the space between the clusters is done by using a Wigner-Seitz type definition, assigning each grid point to the cluster that it is closest to. In the integration in the denominator, we integrate over the whole calculation cell.

If the CTR index is zero, the charge oscillations are happening only inside the clusters, and no net charge is transferred between different clusters. The maximum value of the index is 1 , which would mean that all the induced density oscillations happen between clusters. The absolute value in the numerator makes sure that the index is always positive, since the magnitude is the measurement of the amount of charge transfer between clusters.

\section{RESULTS}

\section{A. Binding energies and HOMO-LUMO gaps}

The binding energies $E_{b}$ per cluster and the HOMO-LUMO (H-L) gaps for the different shapes and separations are listed in Table I, as are the abbreviations given to each system. The values for two systems of dimers consisting of the same eight-electron clusters are also included for comparison. The binding energy for the cluster arrangement is calculated as $E_{b}=M E_{\text {single }}-E_{\text {sys }}$, where $M$ is the number of clusters in the arrangement, $E_{\text {single }}$ the energy of the single cluster, and $E_{s y s}$ the ground-state energy of the cluster system.

We can see that all the system have relatively large H-L gaps close to $1 \mathrm{eV}$, as a consequence of the magic nature of the spherical eight-electron cluster. A single, isolated cluster has a H-L gap of $1.50 \mathrm{eV}$. The H-L gaps and binding energies per cluster are quite similar for the different geometries. The arrangements with 12 clusters have stronger binding energies per cluster than the systems with other sizes, with the icosahedra having the biggest energies for both separations. This trend in the binding energies follows the number of nearest-neighbor interactions per cluster in the different geometries, the tetrahedra and dodecahedra having three, the cuboctahedra four, and the icosahedra five nearest neighbors for each cluster.

Decreasing the separation between the clusters from $S / r=0.5$ to $S / r=0.25$ has the same trend for each threedimensional shape: the binding energy per cluster increases by $0.12-0.20 \mathrm{eV}$, and the H-L gap decreases by $0.19-0.32 \mathrm{eV}$. The changes in the H-L gap can be explained by the further hybridization and subsequent splitting of the electron orbitals of the individual clusters when the clusters are brought closer together.

\section{B. Absorption spectra and CTR values}

The absorption spectra and the CTR values for all the systems are shown in Fig. 3. The absorption strengths have been normalized by dividing by the number of clusters for each system. The spectrum of a single eight-electron cluster is shown for comparison. The clusters on the left side of the cluster arrangement, included in the CTR calculations using Eq. (3), are 1 for the tetrahedra, 1-4 for the icosahedra, 1-4 for the cuboctahedra, and 1-8 for the dodecahedron, using the numbering from Fig. 1.

The spectra shown are the average for light polarized in the $x, y$, and $z$ directions. The cluster arrangements are highly symmetric, and the spectra were checked to be almost identical for all the directions. The same has been observed for the scattering spectra of tetrahedral and icosahedral superclusters of larger nanoparticles both experimentally and computationally [9].

For the larger separation, the $I h l$ in Fig. 3(f) and $C h l$ in Fig. $3(\mathrm{~g})$ both have one relatively sharp main peak at the energies $2.61 \mathrm{eV}$ and $2.62 \mathrm{eV}$, respectively. This peak is, however, broader than and slightly red-shifted from the LSPR peak of the single eight-electron cluster, at $2.72 \mathrm{eV}$, as seen in Fig. 3(h). The red-shift and broadening of the plasmon peak in the absorption spectra also has been observed in experiments of plasmonic NP arrangements [10-13]. The spectrum of Th1, however, supports a broad peak at $2.75 \mathrm{eV}$ with a shoulder at approximately $2.5 \mathrm{eV}$. This is because, on average, the clusters in the tetrahedral arrangement are closer to each other than in the other geometries, which have a larger empty space in the middle. For all the cluster arrangements, the spectrum has a low-energy tail that is absent for the single sphere.

We can observe that going from the larger separation to the smaller, the main peak broadens and absorption strength is transferred to smaller energies, as has been seen also for the dimer systems [21]. This is clearer for the icosahedral and cuboctahedral symmetries, which support quite a narrow peak for $S / r=0.5$, than for the tetrahedra. In these systems, we can see that the optical spectra change drastically with respect to changes in the intercluster separation. For $S / r=0.25$, the highest peak appears at slightly higher energies than for $S / r=$ 0.5 for all the geometries.

Comparing the two different geometries with the same number of clusters, icosahedron and cuboctahedron, we can see that the spectra are very similar for the larger separation. For $S / r=0.25$, the spectra, while still similar, differ somewhat in the details. One explanation is the splitting of the orbitals, which is similar for $C h l$ and $I h l$, but larger for $C h 2$ than for Ih2, as seen in Figs. 4 and 5. The larger splitting 

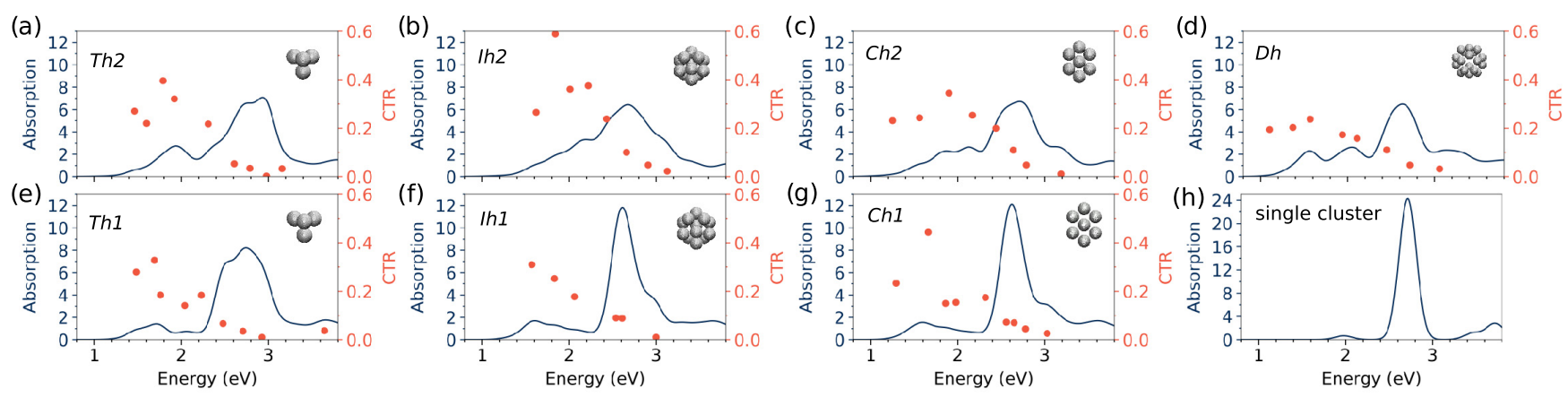

FIG. 3. The absorption spectra and CTR values for all the studied systems. The absorption strength has been divided by the number of clusters for each system. The spectrum of a single cluster, shown with a different $y$-axis scale, is included for comparison.

can be explained by the lower symmetry of the cuboctahedral shape.

Following the CTR analysis, we can see that all the systems have high CTR values at low energies, approximately up to $2 \mathrm{eV}$. The CTR index reaches its minimum at about $3 \mathrm{eV}$, approximately the energy of the higher-energy shoulder of the main peak. This indicates that the low-energy excitations have a high charge transfer character, and the largest peak and its shoulder are a result of charge oscillations inside each cluster separately.

The increase in the CTR values going from the larger separation to the smaller are not very high, with $C h l$ even having one higher CTR values than Ch2. However, for the tetrahedra and the icosahedra, the highest CTR value for $S / r=0.25$ is larger than for $S / r=0.5$ and appears at higher energies. For all the studied systems with two different separations, the CTR values diminish faster when going to bigger energies for the larger separation, which can be seen for example from the data points near $2 \mathrm{eV}$ in Fig. 3.

\section{Analysis of the absorption peaks}

In Fig. 4 we show the DTCMs and induced transition densities for three selected excitations for both of the icosahedral clusters. The main trends with respect to the energy of the excitations are representative also of the three other geometries. The analysis was made for light polarized in the $x$ direction. The contour plot shows the relative strengths of the positive (red) and negative (blue) contributions to the dipole moment. The dashed line marks $e-h$ transitions from initial K-S state $i$ to final state $f$, with energies $\epsilon_{i}$ and $\epsilon_{f}$, respectively, for which the energy of the excitation is $E=\epsilon_{f}-\epsilon_{i}$.

The low-energy excitations shown in Figs. 4(a), 4(b), 4(d), and 4(e) have a high CTR value, the highest for Ihl being the one at $1.57 \mathrm{eV}$ and for $I h 2$ the one at $1.84 \mathrm{eV}$. The isosurfaces of the induced densities show that the induced charge distributions inside one cluster are different on the left and on the right side of the arrangement, clusters on the left being more positively charged. From the DTCMs we can see that the contributions to these excitations below $2 \mathrm{eV}$ are mainly from transitions from the $1 \mathrm{P}$ orbitals to the $1 \mathrm{D}$ orbitals.

The higher-energy excitations, at $2.61 \mathrm{eV}$ and $2.66 \mathrm{eV}$, correspond to the largest peak and have a low CTR value. From the induced density figures we can see that these ex- citations correspond to the electronic charge oscillating inside each cluster separately in-phase. These features indicate that these peaks are bonding combinations of the LSPR peaks. In addition to the $1 \mathrm{P} \rightarrow 1 \mathrm{D}$ transitions, there are also some appreciable contributions from $1 \mathrm{P} \rightarrow 2 \mathrm{~S}$.

The other clear difference between the high- and low-CTR excitations is the ratio of positive and negative contributions to the dipole moment. The percentage of the total strength from the negative contributions of the total strength from all the contributions is largest for the lowest-energy excitations and decreases with increasing energy at least until about $2.5 \mathrm{eV}$. This percentage for $I h l$ is $34 \%, 31 \%$, and $6 \%$, for the peaks shown in Figs. 4(a), 4(b), and 4(c), respectively. Similarly, for $I h 2$, the percentages are $35 \%$ for the peak in Fig. 4(d), 30\% for Fig. 4(e), and 8\% for Fig. 4(f). Thus, the low-energy excitations exhibiting a large CTR have relatively more contributions from screening transitions than the excitations close to the original LSPR peak. These transitions contributing negatively lie mostly on the $E=\epsilon_{f}-\epsilon_{i}$ line, meaning that the energy of the transitions is approximately the energy of the whole excitation. In contrast, the strongest positive excitations lie mostly below this line.

The DTCMs and induced densities for the peak with the highest CTR value for the rest of the systems with $S / r=$ $0.25 r, T h 2, C h 2$, and $D h 2$, can be seen in Fig. 5. The features are similar to those for the peaks with highest CTR values for the icosahedral systems. The cuboctahedron, which has the most splitting in the 1P orbitals of all the geometries, has consequently the biggest spread of energy differences $\epsilon_{f}-\epsilon_{i}$ in the contributing e-h transitions. The largest splitting can be explained by the cuboctahedron having lower symmetry than the icosahedron and the dodecahedron, and having more clusters than the tetrahedron.

We can see strong negative contributions approximately on the $E=\epsilon_{f}-\epsilon_{i}$ line also for all the excitations in Fig. 5. The percentages of the negative $e-h$ contributions are $29 \%$ for the excitation in Fig. 5(a), 26\% for Fig. 5(b), and 30\% for Fig. 5(c). The induced densities again show that the temporary induced charge distributions are not symmetric with respect to all the single clusters, only with respect to the whole arrangement. These features are similar to those for the CTP excitations for dimers of the eight-electron clusters with separations of $S / r=0.5 r$ and $S / r=0$ [21], which also appear in the same energy range between approximately 1.5 and $2 \mathrm{eV}$. Using the same terminology, the excitations seen in 

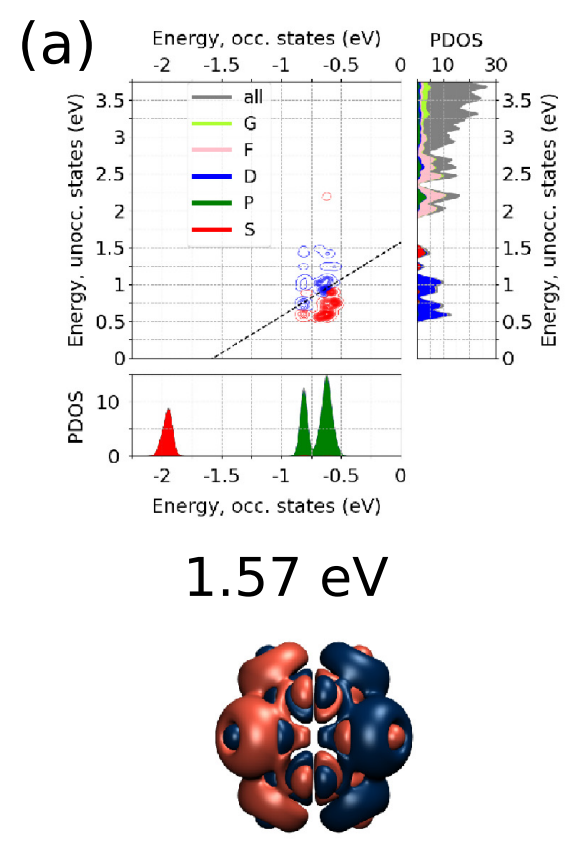

(b)
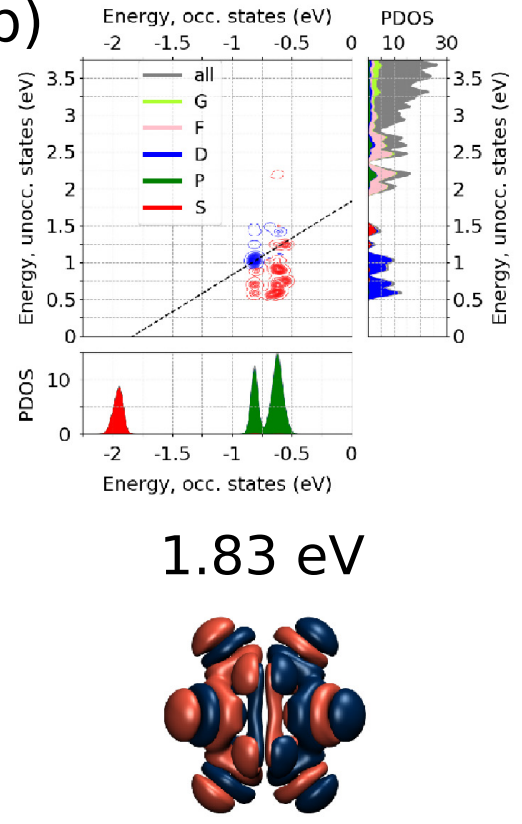

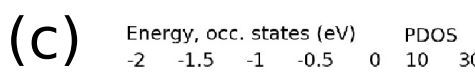
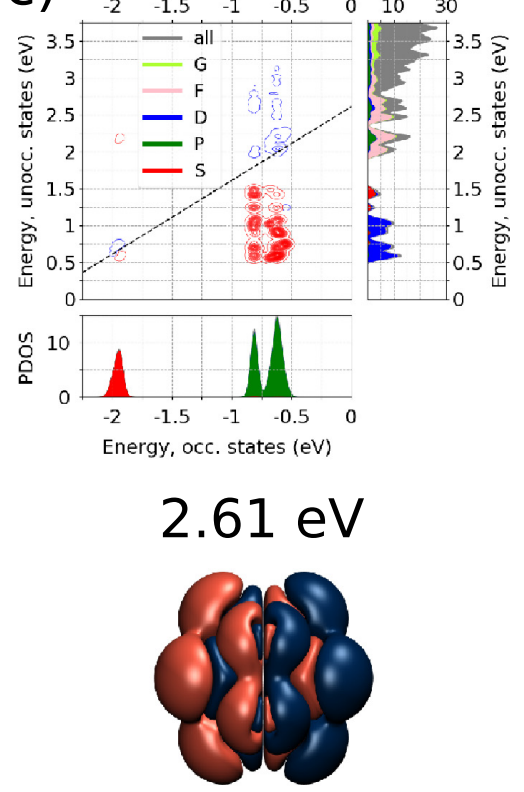

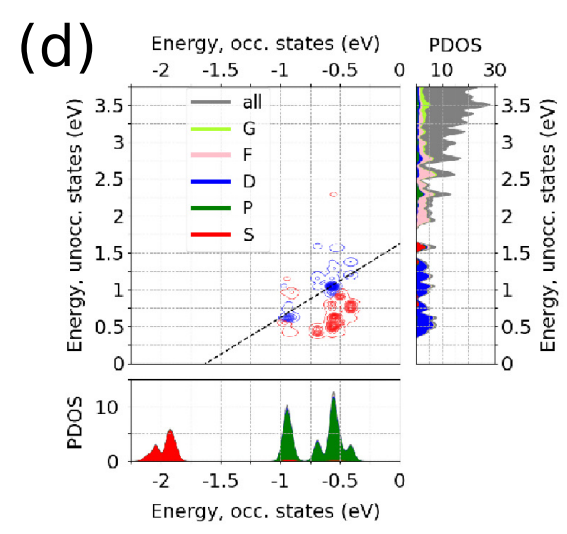

$1.62 \mathrm{eV}$

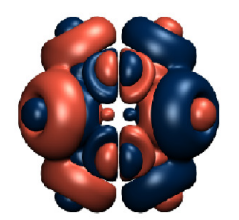

(e)

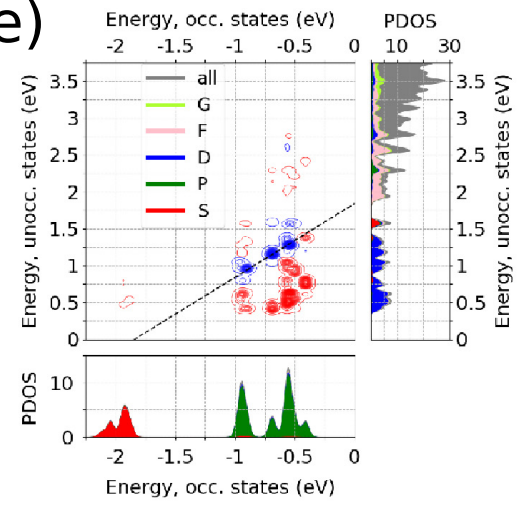

$1.84 \mathrm{eV}$

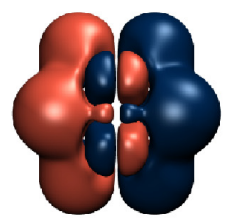

(f)

f) Energy, occ. states (ev) PDOs

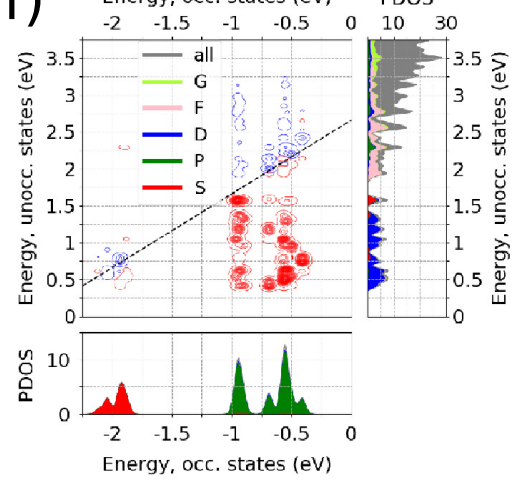

$2.66 \mathrm{eV}$

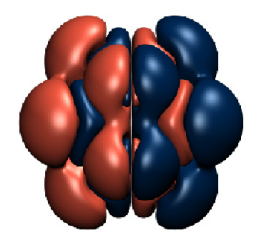

FIG. 4. The DTCM figures and isosurfaces of induced densities for selected peaks for the icosahedral geometries. (a-c) Separation $S=0.5 r$ (Ih1), (d)-(f): separation $S=0.25 r$ (Ih2). The red and blue of the contour plot show positive and negative contributions to the transitions dipole moment, respectively. The colors of the PDOS indicate the superatomic symmetries of the orbitals around each individual cluster in the arrangement, and the PDOS shown is the sum of these projections. The gray indicates higher symmetries and electron density outside of the projection spheres.

Figs. 4(a), 4(b), 4(d), and 4(e) and Fig. 5 can also be classified as CTPs.

\section{SUMMARY AND CONCLUSIONS}

We have studied the plasmonic excitations in geometric assemblies of eight-electron jellium clusters using lr-TDDFT. We have also calculated the CTR index, a measure of the charge transfer nature of the excitations, at different excita- tion energies for each cluster. With the studied separations of approximately 1 or $2 \AA$, even a $1 \AA$ change can have a big effect on the spectra. However, we found excitations with CTP character at low energies for both separations.

For all the studied geometries, we see the same trends in the optical response. The two arrangements with the same number of clusters but different geometries, icosahedron and cuboctahedron, have very similar spectra especially for the longer separation. In addition, all the clusters support one 

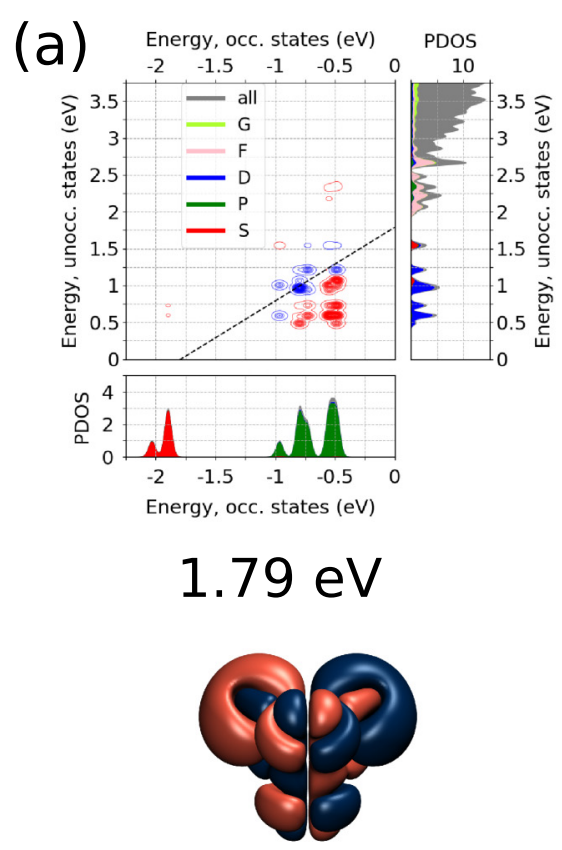

(b)
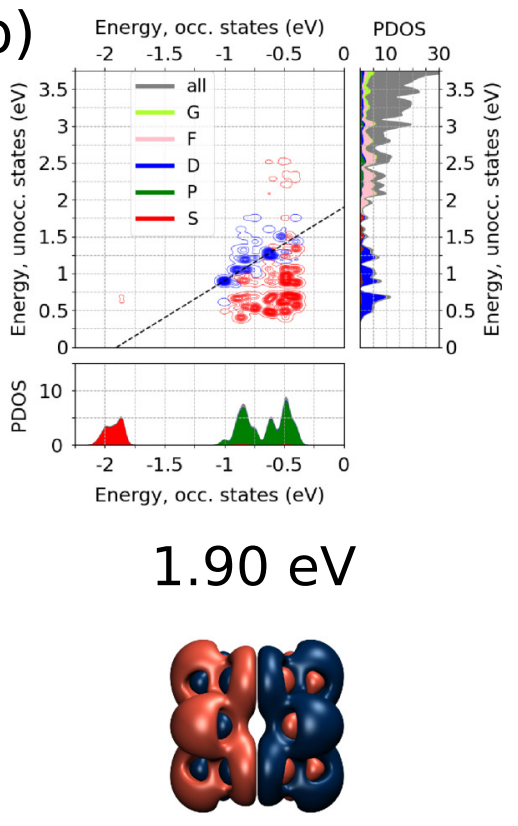
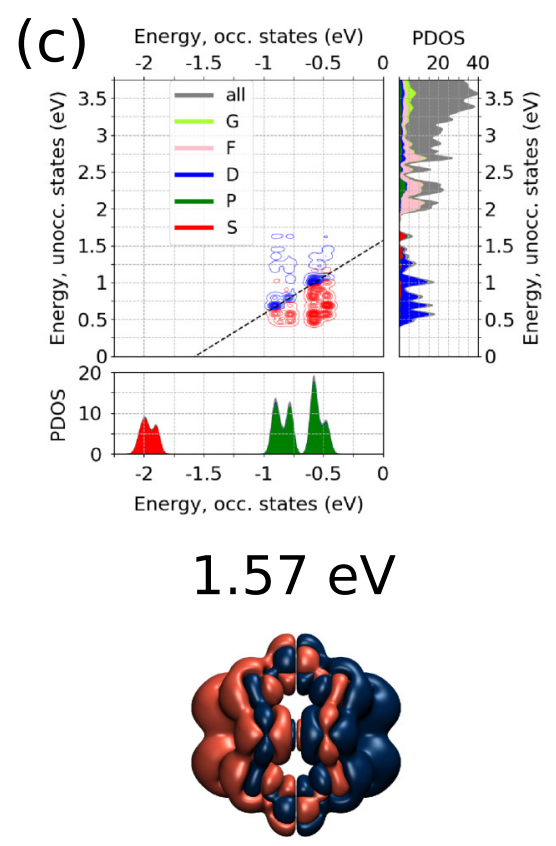

FIG. 5. The DTCM figures and isosurfaces of induced densities for the peak with the highest CTR value for (a) Th2, (b) $C h 2$, and (c) $D h$, which all have a separation of $S=0.25 r$.

larger bonding plasmon peak (in some cases with a shoulder), and one or several CTP peaks at lower energies.

The different nature of the excitations can be also seen in the DTCM analysis: for the peaks with significant charge transfer nature, the $e-h$ transitions contributing to the transition dipole moment are mainly from $1 \mathrm{P}$ to $1 \mathrm{D}$, and they involve transitions with strong negative contributions. The plasmon peaks at higher energies, on the other hand, are formed mainly from positively contributing transitions and include also $1 \mathrm{P} \rightarrow 2 \mathrm{~S}$ contributions.

The combination of the GPAW code and the simple jellium model lends itself readily to the study of different cluster arrangements. The geometries studied here are regular polyhedra. Since GPAW is a real-space grid-based code, any arrangement of clusters is easy to assemble and compute. The same methods and analysis tools can be extended to more complicated or larger geometries as well as clusters of different shapes. Conductive linking between the clusters can also be modeled by channels of suitable jellium background density.

Compared to real eight-electron sodium clusters, the uniform spherical jellium background is an oversimplification. For example, since atomic $\mathrm{Na}_{8}$ clusters are polygons in shape, the relative orientations of the neighboring clusters can also affect the charge transfer and plasmon coupling in an assembly. The effect of the shape could be studied by changing the jellium background from spherical to the experimental geometry, which would be an interesting follow-up work. Here we concentrated on the general trends varying the separation and arrangement, the results representing rather an assembly of different cluster orientations than a single atomic system.

In the experimental studies and applications, the clusters usually consist of gold or silver atoms, not sodium. In these noble metals, the $d$ electron screening weakens the surface plasmon resonance and thus reduces the interaction between the plasmons of neighboring clusters [33]. Additionally, gold and silver have smaller Wigner-Seitz radii than sodium, resulting in larger background density. This corresponds to smaller electron spill-out lengths for these noble metals [34,35]. Since the CTPs require electron density overlap, this indicates that the onset of the CTPs would happen at smaller separation in gold or silver arrangements. Because of these differences, noble metal assemblies are expected to show a weaker coupling in the absorption spectra than the jellium arrangements with the density of sodium studied here.

Exact comparison to existing experiments is also hindered by the generally much larger size of the NPs and interparticle separations in the experimental plasmonic assemblies, such as those mentioned in the introduction, compared to the jellium arrangements studied here. Specifically, none of the articles [5-13] report CTPs, which require very small intercluster separations. So far, CTPs have not been observed for cluster systems with similar arrangements and cluster sizes as studied here. Atomically precise monolayer-protected metal clusters [36] are promising candidates for building blocks of assemblies that would enable the experimental study of the plasmon coupling and charge transfer oscillations in this size range.

The jellium studies can pave the way to more realistic atomistic calculations for modeling these systems. For example, even though the induced densities and DTCMs are more complex for assemblies of monolayer-protected gold clusters, the trends can be expected to be the same as here. By comparing the results of jellium calculations and atomistic calculations, the effect of the atomic structure can be separated. In addition, the results for jellium assemblies can help to identify the different features in the optical spectra for the atomistic systems. 


\section{ACKNOWLEDGMENTS}

The authors thank Mikael Kuisma for useful discussions. This work was supported by the Academy of Finland (Grants No. 294217 and No. 319208, H.H.'s Academy Professorship), the Emil Aaltonen Foundation, and the Finnish Cultural Foundation (E.S.'s Ph.D. scholarships). The computations were done at the CSC, the Finnish IT Center for Science (Grand Challenge project COUPLES).
[1] E. Prodan, C. Radloff, N. J. Halas, and P. Nordlander, A hybridization model for the plasmon response of complex nanostructures, Science 302, 419 (2003).

[2] L. Polavarapu, J. Pérez-Juste, Q.-H. Xu, and L. M. LizMarzán, Optical sensing of biological, chemical and ionic species through aggregation of plasmonic nanoparticles, J. Mater. Chem. C 2, 7460 (2014).

[3] G. Haran and L. Chuntonov, Artificial plasmonic molecules and their interaction with real molecules, Chem. Rev. 118, 5539 (2018).

[4] J. Zuloaga, E. Prodan, and P. Nordlander, Quantum description of the plasmon resonances of a nanoparticle dimer, Nano Lett. 9, 887 (2009)

[5] S. J. Barrow, X. Wei, J. S. Baldauf, A. M. Funston, and P. Mulvaney, The surface plasmon modes of self-assembled gold nanocrystals, Nat. Commun. 3, 1 (2012).

[6] L. Lermusiaux and A. M. Funston, Plasmonic isomers via DNA-based self-assembly of gold nanoparticles, Nanoscale 10, 19557 (2018).

[7] K. J. Park, J.-H. Huh, D.-W. Jung, J.-S. Park, G. H. Choi, G. Lee, P. J. Yoo, H.-G. Park, G.-R. Yi, and S. Lee, Assembly of â3Dâ plasmonic clusters by â2Dâ AFM nanomanipulation of highly uniform and smooth gold nanospheres, Sci. Rep. 7, 1 (2017).

[8] N. Pazos-Perez, C. S. Wagner, J. M. Romo-Herrera, L. M. Liz-Marzán, F. J. García de Abajo, A. Wittemann, A. Fery, and R. A. Alvarez-Puebla, Organized plasmonic clusters with high coordination number and extraordinary enhancement in surface-enhanced Raman scattering (SERS), Angew. Chem. Int. Ed. 124, 12860 (2012).

[9] A. S. Urban, X. Shen, Y. Wang, N. Large, H. Wang, M. W. Knight, P. Nordlander, H. Chen, and N. J. Halas, Threedimensional plasmonic nanoclusters, Nano Lett. 13, 4399 (2013).

[10] X. Huang, Y. Liu, J. Barr, J. Song, Z. He, Y. Wang, Z. Nie, Y. Xiong, and X. Chen, Controllable self-assembled plasmonic vesicle-based three-dimensional SERS platform for picomolar detection of hydrophobic contaminants, Nanoscale 10, 13202 (2018).

[11] J. Fontana, W. J. Dressick, J. Phelps, J. E. Johnson, R. W. Rendell, T. Sampson, B. R. Ratna, and C. M. Soto, Virus-templated plasmonic nanoclusters with icosahedral symmetry via directed self-assembly, Small 10, 3058 (2014).

[12] N. Lebedev, I. Griva, W. J. Dressick, J. Phelps, J. E. Johnson, Y. Meshcheriakova, G. P. Lomonossoff, and C. M. Soto, A virusbased nanoplasmonic structure as a surface-enhanced Raman biosensor, Biosens. Bioelectron. 77, 306 (2016).

[13] H. A. Nguyen, I. Jupin, P. Decorse, S. Lau-Truong, S. Ammar, and N. T. Ha-Duong, Assembly of gold nanoparticles using turnip yellow mosaic virus as an in-solution SERS sensor, RSC Adv. 9, 32296 (2019).
[14] Y. A. Urzhumov, G. Shvets, J. Fan, F. Capasso, D. Brandl, and P. Nordlander, Plasmonic nanoclusters: A path towards negativeindex metafluids, Opt. Express 15, 14129 (2007).

[15] T. L. Doane and C. Burda, The unique role of nanoparticles in nanomedicine: Imaging, drug delivery and therapy, Chem. Soc. Rev. 41, 2885 (2012)

[16] A. G. Skirtach, C. Dejugnat, D. Braun, A. S. Susha, A. L. Rogach, W. J. Parak, H. Möhwald, and G. B. Sukhorukov, The role of metal nanoparticles in remote release of encapsulated materials, Nano Lett. 5, 1371 (2005).

[17] T. S. Troutman, S. J. Leung, and M. Romanowski, Lightinduced content release from plasmon-resonant liposomes, Adv. Mater. 21, 2334 (2009).

[18] Nonappa, T. Lahtinen, J. S. Haataja, T.-R. Tero, H. Häkkinen, and O. Ikkala, Template-free supracolloidal self-assembly of atomically precise gold nanoclusters: From 2D colloidal crystals to spherical capsids, Angew. Chem. Int. Ed. 128, 16269 (2016).

[19] V. Marjomäki, T. Lahtinen, M. Martikainen, J. Koivisto, S. Malola, K. Salorinne, M. Pettersson, and H. Häkkinen, Site-specific targeting of enterovirus capsid by functionalized monodisperse gold nanoclusters, Proc. Natl. Acad. Sci. USA 111, 1277 (2014)

[20] E. Selenius, S. Malola, and H. Häkkinen, Analysis of localized surface plasmon resonances in spherical jellium clusters and their assemblies, J. Phys. Chem. C 121, 27036 (2017).

[21] E. Selenius, S. Malola, M. Kuisma, and H. Häkkinen, Charge transfer plasmons in dimeric electron clusters, J. Phys. Chem. C 124, 12645 (2020).

[22] V. Bonačić-Kouteck, P. Fantucci, and J. Kouteck, Systematic $a b$ initio configuration-interaction study of alkali-metal clusters. II. Relation between electronic structure and geometry of small sodium clusters, Phys. Rev. B 37, 4369 (1988).

[23] I. A. Solovâyov, A. V. Solovâyov, and W. Greiner, Structure and properties of small sodium clusters, Phys. Rev. A 65, 053203 (2002).

[24] P. Zhang, J. Feist, A. Rubio, P. García-González, and F. J. García-Vidal, $A b$ initio nanoplasmonics: The impact of atomic structure, Phys. Rev. B 90, 161407(R) (2014).

[25] J. J. Mortensen, L. B. Hansen, and K. W. Jacobsen, Real-space grid implementation of the projector augmented wave method, Phys. Rev. B 71, 035109 (2005).

[26] J. Enkovaara, C. Rostgaard, J. J. Mortensen, J. Chen, M. Dułak, L. Ferrighi, J. Gavnholt, C. Glinsvad, V. Haikola, H. A. Hansen et al., Electronic structure calculations with GPAW: A real-space implementation of the projector augmented-wave method, J. Phys.: Condens. Matter 22, 253202 (2010).

[27] J. P. Perdew and Y. Wang, Accurate and simple analytic representation of the electron-gas correlation energy, Phys. Rev. B 45, 13244 (1992).

[28] M. Walter, H. Häkkinen, L. Lehtovaara, M. Puska, J. Enkovaara, C. Rostgaard, and J. J. Mortensen, Time-dependent 
density-functional theory in the projector augmented-wave method, J. Chem. Phys. 128, 244101 (2008).

[29] X. Andrade, S. Botti, M. A. Marques, and A. Rubio, Timedependent density functional theory scheme for efficient calculations of dynamic (hyper)polarizabilities, J. Chem. Phys. 126, 184106 (2007).

[30] S. Malola, L. Lehtovaara, J. Enkovaara, and H. Häkkinen, Birth of the localized surface plasmon resonance in monolayerprotected gold nanoclusters, ACS Nano 7, 10263 (2013).

[31] T. P. Rossi, M. Kuisma, M. J. Puska, R. M. Nieminen, and P. Erhart, Kohn-Sham decomposition in real-time time-dependent density-functional theory: An efficient tool for analyzing plasmonic excitations, J. Chem. Theory Comput. 13, 4779 (2017).
[32] M. Walter, J. Akola, O. Lopez-Acevedo, P. D. Jadzinsky, G. Calero, C. J. Ackerson, R. L. Whetten, H. Grönbeck, and H. Häkkinen, A unified view of ligand-protected gold clusters as superatom complexes, Proc. Natl. Acad. Sci. USA 105, 9157 (2008).

[33] D. W. Brandl, C. Oubre, and P. Nordlander, Plasmon hybridization in nanoshell dimers, J. Chem. Phys. 123, 024701 (2005).

[34] N. Lang and W. Kohn, Theory of metal surfaces: Charge density and surface energy, Phys. Rev. B 1, 4555 (1970).

[35] N. Lang and W. Kohn, Theory of metal surfaces: Work function, Phys. Rev. B 3, 1215 (1971).

[36] T. Tsukuda and H. Häkkinen, Protected Metal Clusters: From Fundamentals to Applications, Frontiers in Nanoscience Vol. 9 (Elsevier, Amsterdam, 2015). 